
FEASIBILITY AND TECHNICAL NOTE OF INITIAL EXPERIENCE

L Ponce Mejia*. UCLA, Los Angeles, CA

10.1136/neurintsurg-2021-SNIS.24

Background Preliminary experiences of robotic assisted neuroendovascular interventions have been recently reported. We aim to describe the feasibility and our initial experience of robotic-assisted neuroangiography for diagnosis and treatment planning.

Objective To discuss our initial experience, feasibility and technical note in our early experience with the use of the CorPath GRX robotic-assisted technology for neuroendovascular procedures, including transfemoral, transradial and distal transradial diagnostic neuroangiograms.

Methods Single-center technical report of the first fortyfour consecutive cases of robtic assisted diagnostic neuroangiography using the CorPath GRX Robotic System (Corindus Inc, Waltham, MA) from October 2020 through February 2021.

Results 43 diagnostic neuroangiographies were planned. In 38 cases, at least 1 vessel was selected and studied using the CorPath GRX robotic-assisted technology, in two cases the neuroangiography was completed by switching to manual, allowing for 36 diagnostic neuroangiograms to be fully completed as planned using the CorPath GRX robotic-assisted technology. In five $(11 \%)$ cases, the robot was setup but it was either not used due to a change in plans, or inability to select at least one single vessel as planned. In one case, failure was due to defective cassette and in another case due to communication error between joystick and robotic arm. [NK1] The mean of number or vessels successfully selected and studied using robotic assisted neuroangiography was 4.1 (ranging from 1 to 7 for cerebral angiograms, and 22 for one spinal angiogram). No complications were reported as result of the use the CorPath GRX robotic technology to perform neuroangiograms. The mean fluoroscopy time for the 23 cerebral angiograms was 16.5 minutes [8.9 - 27.2 minutes], and the mean radiation exposure to the patients was $63.1 \mathrm{~Gy} / \mathrm{cm}^{2}\left[30.3-105 \mathrm{~Gy} / \mathrm{cm}^{2}\right]$. [NK2] Catheterization failures involved mostly wire slipping and catheter slipping. Simmon-2 shape catheter were did not pose a particular challenge when being formed. Our group quickly learned that the V18 was the microwire of choice as well the $5 \mathrm{~F}$ Terumo XP version of each catheter tip shape to maximize catheterization success. An additional $0.018^{\prime}$ companion wire was used successfully for additional support when advancing 6F guide sheaths from the aortic arch into the innominate artery and common carotid artery.

Conclusions We describe our initial experience of the CorPath GRX system (Corindus) to navigate the arch and great vessels, and in 1 case the spinal segmental vessels in patients as clinically indicated. We also demonstrated the feasibility of completing the diagnostic portion of our planned endovascular embolization cases, thus minimizing radiation exposure and the need to wear apron lead to the operators.

Disclosures L. Ponce Mejia: None.

\section{0-025 TECHNICAL OPTIONS TO OBTAIN COMPLETE OBLITERATION OF VEIN OF GALEN ANEURYSMAL MALFORMATION}

T Shigematsu*, J Fifi, A Berenstein. Cerebrovascular Center, Department of Neurosurgery, Mount Sinai Health System, New York, NY

\subsection{6/neurintsurg-2021-SNIS.25}

Background Since staged trans-arterial endovascular embolization (TAE) became the main strategy to treat vein of Galen aneurysmal malformation (VGAM), this otherwise lethal disease has become manageable. The antenatally diagnosed cases need proper planning for delivery and neonatal management. Symptomatic neonates with congestive heart failure (CHF) benefit from trans-umbilical TAE with closure of fistulas in order to reduce CHF and improve survival. In infants, symptomatic hydrocephalus or venous hypertension requires urgent TAE to facilitate normal brain development. Even in asymptomatic cases, staged TAE in a safe manner can be beneficial before development delay. Staged TAE of the choroidal feeding arteries has been reported to result in cure. However, some VGAMs remain technically challenging to obtain complete obliteration solely with this technique. If the choroidal feeders are not accessible after prior TAE, other options need to be sought. We reviewed the final few procedures in consecutively cured VGAM patients and assessed the techniques utilized to obtain total obliteration.

Materials and Methods This is a retrospective chart and radiographic investigation of a consecutive series of 124 VGAM cases treated between 2004 and 2021 in our institution. Obvious arteriovenous malformation with aneurysmal dilatation of vein of Galen was not included. The strategy in this period was to repeat TAE of the choroidal feeders until the VGAM was totally occluded or until choroidal feeder access was no longer safe. In 81 out of 124 patients, the VGAM was totally or near totally $(>95 \%)$ obliterated. There were 7 death because of the SHF instead of aggressive TAE or because of the hemorrhagic complication. 36 patients are currently undergoing staged embolization and total obliteration hasn't been achieved and are therefore excluded from this study.

Results Among the 81 cases, 66 cases (81\%) achieved total to near total obliteration of the VGAM solely with staged TAE through choroidal artery or thalamoperforating artery feeders. Most of them were found to have total occlusion in the 3 to 6 month follow up angiogram after a final TAE, even when the malformation had been present at the final angiogram in the last TAE. In 4 cases (5\%), the dural supply of the malformation was embolized with ONYX from the external carotid artery as the final procedure; and total obliteration was achieved. Transvenous embolization at the last procedure led to complete occlusion of the VGAM in 9 patients (11\%). Two cases were referred to stereotactic radiosurgery (SRS) after the age of 10 and total obliteration of the residual malformation was confirmed in the 2 year follow up cerebral angiogram post SRS. There was one hemorrhagic complication occurring in a case after final TVE.

Conclusions Staged transarterial n-BCA embolization of the choroidal arteries effected cure of VGAM with a good outcome in more than $80 \%$ of the 124 cases. If the access is 